\section{„Von der Locke bis zur Socke Interventionelle Radiologie“}

Auf dem 97. Deutschen Röntgenkongress in Leipzig (4.-7. Mai 2016) wurde Prof. Dr. Christian Stroszczynski zum neuen Vorsitzenden der Deutschen Gesellschaft für Interventionelle Radiologie und minimal-invasive Therapie (DeGIR) gewählt. Im Interview erläutert er, welche Bedeutung die DeGIR für die Interventionelle Radiologie hat und welche Ziele er sich für seine Amtszeit als Vorsitzender gesteckt hat.

\section{Was zeichnet die Interventionelle Radiologie aus?}

Interventionelle Radiologie ist bildgeführte Therapie. Man nutzt bildgebende Verfahren, um medizinische Instrumente im Körper zu steuern, die man entweder über die Gefäße oder direkt durch die Haut in den Körper einführt. Damit ist die Interventionelle Radiologie zwar ein sehr technikbasierter Bereich, andererseits können damit zahlreiche Erkrankungen direkt behandelt werden. In der Medizin wird zunehmend organbezogen gearbeitet. So gibt es beispielsweise Gefäßzentren, Leberzentren oder Schlaganfallzentren, die auf ein bestimmtes klinisches Symptom fokussieren. Die Interventionelle Radiologie hingegen macht alles von Kopf bis Fuß. Wir behandeln jegliche Erkrankungen und richten unsere Arbeit eher an den unterschiedlichen Modalitäten aus. Der interventionelle Radiologe ist deshalb auch in jedem Fall ein Teamplayer, denn der Patient geht ja nicht auf direktem Wege zum Radiologen oder zum interventionellen Radiologen. Wir sind vielmehr darauf angewiesen, dass wir die Patienten von anderen medizinischen Bereichen zugewiesen bekommen. Wichtig ist deshalb ein Behandlungsteam, das uns aufnimmt und in das wir unsere therapeutischen Kompetenzen und Fertigkeiten einbringen können. Diese Therapien können wie gesagt ganz unterschiedliche Bereiche betreffen - beispielsweise eine Gefäßbehandlung im Rahmen einer peripheren arteriellen Verschlusskrankheit, die Behandlung von Leberkrebs oder von Uterusmyomen. Das macht das Fach so spannend. Daraus resultiert aber auch die Notwendigkeit, der Interventionellen Radiologie eine eigene Stimme zu geben, damit wir in der großen Bandbreite der Behandlungsmöglichkeiten mit unseren Therapien nicht verloren gehen. Wir müssen die Vorteile einer interventionellen radiologischen Behandlung gegenüber anderen Berufsgruppen immer wieder herausheben und einbringen. Interventionellen Radiologen geht es wie Stürmern in einem Fußballspiel. Beide sind darauf angewiesen, dass die Mitspieler den Ball zuspielen. Wenn man jedoch alleine vorne steht und keine Bälle bekommt, kann man auch nichts tun.

\section{Die Interventionelle Radiologie ist doch eine relativ junger Bereich, oder?}

Ja und nein. In der Radiologie wurden schon immer bildgesteuerte Eingriffe vorgenommen. Radiologen haben schon früh versucht, mit einer Nadel, also minimalinvasiv ohne Schnitt, dorthin zu kommen, wo sie auf der Aufnahme etwas entdeckt haben. Die offizielle Geburtsstunde der Interventionellen Radiologie geht auf eine Innovation von Charles Dotter zurück. Dotter hatte 1964 damit begonnen, Gefäße interventionell zu dilatieren. Für lange

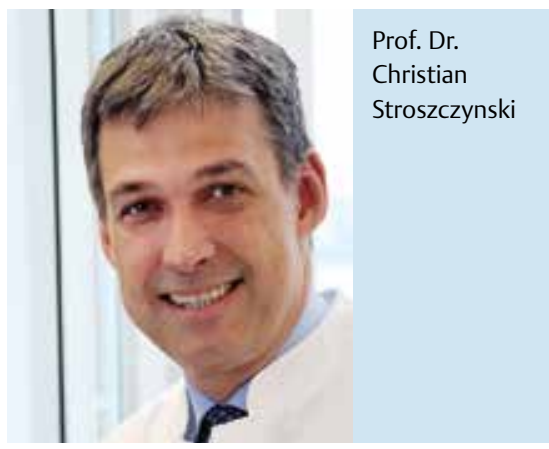

Jahre war die Interventionelle Radiologie damit nahezu gleichbedeutend mit der Angiographie, was nicht zuletzt auch auf die Behandlungsnotwendigkeit der peripheren arteriellen Verschlusskrankheit als Volkskrankheit zurückzuführen ist. Erst in den letzten 20 Jahren sind dann eine Reihe von Eingriffen hinzugekommen, die mit Gefäßerkrankungen nicht mehr viel zu tun haben. In der Behandlung von Leberkrebs zum Beispiel ist die Interventionelle Radiologie momentan ganz vorne mit dabei. Hier haben wir inzwischen sogar mehr Patienten als die Chirurgen oder Internisten. Wir sind also durchaus noch ein sehr junges und dynamisches Fach, da von den heroischen Erstversuchen bis zur heutigen leitliniengerechten Therapie erst gut 50 Jahre vergangen sind. Viele Pioniere von damals leben heute noch und sind teilweise sogar noch beruflich aktiv. 
Warum gibt es überhaupt eine eigene Gesellschaft für Interventionelle Radiologie und minimal-invasive Therapie?

Die DeGIR wurde 2008 gegründet und dies aus der Notwendigkeit heraus, der Interventionellen Radiologie eine eigene Stimme zu geben. In der damaligen Konstellation innerhalb der DRG wurde bzw. konnte die Interventionelle Radiologie nicht in dem Maße gefördert und unterstützt werden, wie es notwendig war, um sich gegenüber den anderen Fachgruppen zu behaupten. Es ist ja auch aktuell wieder so, dass viele andere medizinischen Fächer radiologische und vor allem interventionell radiologische Verfahren für sich beanspruchen. Dem mussten und müssen wir unbedingt entgegenwirken. Interventionelle Radiologen haben nach wie vor den großen Vorteil, dass sie breit aufgestellt und hervorragend ausgebildet sind. Es war und ist daher wichtig, dass wir als eigene Fachgesellschaft nach außen auftreten können und dabei nicht nur als Diagnostiker, sondern auch als gleichberechtigte Behandler wahrgenommen werden. Das ist vor allem bei Fragen der Vergütung, in Strukturfragen und bei Leitlinienvorhaben von immenser Bedeutung.

\section{Welche Ziele verfolgt die DeGIR?}

Ein zentrales Anliegen besteht darin, eine bestmögliche Behandlungsqualität zu gewährleisten. Die interventionellen Radiologen sind diejenigen, die für interventionelle und minimal-invasive Eingriffe am besten ausgebildet sind. Diese Exzellenz muss man auch zeigen und sichtbar belegen können. Der Schlüssel hierzu sind Zertifizierungen. Hier war die DeGIR Vorreiter und hat mit ihrem Zertifizierungssystem die Weichen für viele weitere Zertifizierungen anderer Fachgesellschaften gestellt. Das neueste Projekt sind die DeGIRZentren für interventionelle Gefäßmedi- zin und minimal-invasive Therapie. Mit diesem Siegel dokumentiert man nach außen, dass hier ein Experten-Team sitzt, das von der Locke bis zur Socke Interventionelle Radiologie anbieten kann.

\section{Welche konkreten Pläne haben Sie für Ihre Amtszeit 2016-2018?}

Ich möchte, dass wir für die Patienten und auch für die Fachkollegen besser sichtbar werden. Dies kann durch eine verstärkte Öffentlichkeitsarbeit geschehen, aber auch durch die Schaffung neuer struktureller Möglichkeiten in Kliniken und Praxen. Ich kenne einige vorbildliche Beispiele von Spezialambulanzen in Kliniken, in denen interventionelle Radiologen gleichberechtigt mit Internisten und Chirurgen Patienten betreuen. Das heißt, hier kommen die Patienten direkt zu den interventionellen Radiologen und lassen sich zu Therapien beraten, die dann auch vor Ort durchgeführt werden können. In einigen Häusern gibt es sogar schon eigene Betten für die Interventionelle Radiologie. Dies gilt es auszubauen, denn nur so werden interventionelle Radiologen auch als eigenständige Behandler und patientenführende Ärzte wahrgenommen. Das ist mein Ziel.

\section{Wie sind Sie persönlich eigentlich zur Radiologie gekommen?}

Ich habe zunächst mit der Inneren Medizin begonnen, denn die Radiologie begegnet einem Medizinstudenten während seiner Ausbildung auch heute noch erst recht spät. Durch die Fächer Hämatologie und Onkologie im Arzt im Praktikum, was es damals noch gab, habe ich mich sehr für die klinische Seite der Medizin begeistern können. Und die Radiologie wurde und wird immer klinischer. Auch bei uns in Regensburg durchlaufen alle Radiologen, die bei uns anfangen, zunächst ein halbes Jahr in der Chirurgie, damit sie dort mit den klinischen Aspekten vertraut werden. Wie schreibt man einen Arztbrief, wie rede ich mit dem Patienten, wir kläre ich ihn auf, was ist ein Laborwert - all das ist für die Radiologie sehr wichtig geworden.

\section{Und warum nun explizit Interventio-} nelle Radiologie?

Weil mir, neben dem Diagnostischen, auch das Therapeutische immer sehr gelegen hat. Ich wollte herausfinden, was der Patient hat, damit ich dann im nächsten Schritt die richtige Therapie für ihn finden kann. Deshalb war die Interventionelle Radiologie für mich ideal, weil ich hier beides miteinander verbinden konnte: die Detektivarbeit der Diagnosesuche und die eigentliche Hilfe in Form der richtigen Therapie. Es hat jedoch recht lang gedauert, bis ich die Möglichkeit hatte, interventionell zu arbeiten. Das ist leider auch heute noch oft der Fall. Immer wieder höre ich Klagen, dass sich zu wenige junge Leute für die Interventionelle Radiologie interessieren. Das liegt aber auch oft daran, dass es nicht leicht ist, sich über eine Pflichtrotation hinaus gut ausbilden zu lassen. Hier kann jeder Direktor und jeder Chef etwas tun, damit alle, die daran Interesse haben, auch früh mit der Interventionellen Radiologie in Berührung kommen können. Auch dafür möchte ich mich mit der DeGIR einsetzen.

Vielen Dank für das Gespräch! 